\title{
Características clínico-radiológicas en pacientes con neumonía por SARS-CoV-2, estudio descriptivo en un hospital de
} segundo nivel

Juan J. Gómez-Piña1*, Emma R. González-Veyrand² y Roberto R. López Anguiano

${ }^{1}$ Departamento de Medicina Interna, Hospital de Especialidades Dr. Antonio Fraga Mouret, Centro Médico Nacional La Raza; ${ }^{2}$ Servicio de Imagenología, Hospital General de Zona n. 27 Alfredo Badallo; ${ }^{3}$ Departamento de Enseñanza, Hospital General de Zona n. ${ }^{\circ} 27$ Alfredo Badallo. Instituto Mexicano del Seguro Social, Ciudad de México, México

\section{Resumen}

Introducción: La reacción en cadena de la polimerasa con transcripción inversa en tiempo real (RT-PCR) es el estándar de oro para establecer el diagnóstico de enfermedad por coronavirus 2019 (COVID-19). La tomografía computarizada de tórax (TCT), como complemento diagnóstico, clasifica los hallazgos tomográficos de acuerdo con el sistema CO-RADS (COVID-19 Reporting and Data System). Objetivo: Determinar la sensibilidad y especificidad de la TCT para el diagnóstico de COVID-19. Métodos: Consultamos los resultados de RT-PCR, así como sus respectivas TCT. Todas las TCT se clasificaron de según CO-RADS. Se evaluó sensibilidad y especificidad de la TCT utilizando el resultado de RT-PCR más cercano de TCT como referencia. Resultados: Incluimos 412 pacientes, incluyendo 277 hombres (46-70 años) y 130 mujeres (45-71 años), con 181 pruebas negativas y 231 positivas; obteniendo sensibilidad del 92.15\% y especificidad del 79.32\%. La mortalidad aumentó después de seis días de hospitalización, en el sexo masculino y en CO-RADS 4, 5 y 6 en comparación con CO-RADS 1, 2 y 3. Conclusiones: El diagnóstico temprano es decisivo en el pronóstico de la neumonía SARS CoV 2. Aunque la RT-PCR es el estándar de oro actual, los falsos negativos son frecuentes, por lo que la TCT ayuda a confirmar los casos sospechosos, incluso en etapas tempranas. Este estudio de imagen es un estudio accesible y fundamental para la clasificación, diagnóstico y pronóstico.

PALABRAS CLAVE: CO-RADS. Clínico. Radiológico. Características. SARS-CoV-2.

\section{Clinical and radiological characteristics in patients with SARS-CoV-2-associated pneumonia. A descriptive study in a secondary care center}

\begin{abstract}
Introduction: Real-time reverse-transcription polymerase chain reaction (RT-PCR) is the gold standard for establishing the diagnosis of coronavirus disease 2019 (COVID-19). Chest computed tomography (CCT), as a diagnostic complement, classifies tomographic findings according to the COVID-19 Reporting and Data System (CO-RADS). Objective: To determine CCT sensitivity and specificity for COVID-19 diagnosis. Methods: We reviewed RT-PCR results, as well as their respective CCTs. All CCTS were classified according to CO-RADS. CCT sensitivity and specificity were evaluated using the RT-PCR result that was closest to that of CCT as reference. Results: We included 412 patients, out of whom 277 were males (46-70 years) and 130 were females (45-71 years); with 181 negative and 231 positive tests, a sensitivity of $92.15 \%$ and specificity of $79.32 \%$ were obtained. Mortality increased after six hospitalization days, in males and in CO-RADS 4, 5 and 6 in comparison with CO-RADS 1, 2 and 3. Conclusions: Early diagnosis play a decisive role in prognosis of SARS CoV 2 pneumonia; although RT-CPR is the current gold
\end{abstract}

Correspondencia:

*Juan J. Gómez-Piña

E-mail: drjgomezp@gmail.com
Gac Med Mex. 2021;157:594-599

Disponible en PubMed

www.gacetamedicademexico.com

0016-3813/৫ 2021 Academia Nacional de Medicina de México, A.C. Publicado por Permanyer. Este es un artículo open access bajo la licencia CC BY-NC-ND (http://creativecommons.org/licenses/by-nc-nd/4.0/). 
standard, false negatives are common findings, reason why chest CT help to confirm suspected cases, even in early stages. This image study is accessible and a fast tool. Therefore, CT most be considered as an essential study to be done in all suspected COVID 19 patients, classifying into the CO-RADS system classification, as a diagnosis and prognostic tool.

KEY WORDS: CO-RADS. Clinical. Radiological. Characteristics. SARS-CoV-2.

\section{Introducción}

El 31 de diciembre de 2019 en la ciudad de Wuhan, China, se reconoce un brote de neumonía atípica provocada por el coronavirus 2 del síndrome respiratorio agudo grave (SARS-CoV-2), cobrando a lo largo de este año un gran impacto en la supervivencia de la población, ya que se ha manifestado como una pandemia, amenazante para la salud y seguridad pública. Por ser un virus con tropismo respiratorio genera síndrome de dificultad respiratoria, presentándose con mayor frecuencia en pacientes diagnosticados con diabetes mellitus, hipertensión arterial, obesidad y síndrome metabólico, afectando en gran medida a la población mexicana, siendo uno de los virus más mortíferos en nuestro medio. El SARSCoV-2 causa infección de neumocitos, células dendríticas y macrófagos, induciendo la replicación, especialmente en el parénquima donde se ubica el receptor de la enzima convertidora de angiotensina (ACE) II, uniendo el virus al epitelio y comenzando su infección'.

Se han encontrado algunas características del SARS-CoV-2; sin embargo, las características clínicas se han destacado con la presencia de tres episodios clínicos: infección respiratoria leve, neumonía con evidencia de tomografía torácica y neumonía grave².

Ante la rápida introducción de la infección, se han utilizado estudios auxiliares para evidenciar neumonía, siendo la tomografía torácica el mejor estudio de imagen para identificar enfermedad por coronavirus 2019 (COVID-19), incluso características clínicas anteriores, hasta dos semanas después de esto ${ }^{3}$.

Se ha realizado una clasificación radiológica basada en los hallazgos de tomógrafo torácico de COVID-19, clasificándola en cinco etapas según la evolución: ultradesarrollo, progresión temprana y rápida, consolidación y disipación. La primera etapa ocurre entre la exposición tras la primera y segunda semana, en pacientes asintomáticos con consolidaciones, nódulos pulmonares rodeados de vidrio pulido y broncograma. La segunda etapa ocurre de uno a dos días después de los síntomas, con lesiones simples o múltiples, así como con el engrosamiento del tabique interlobulillario.
La tercera etapa ocurre una semana después de los síntomas, con consolidaciones y broncograma. La cuarta etapa ocurre después de la segunda semana de síntomas, con reducción de tamaño y densidad de las consolidaciones pulmonares. La quinta etapa ocurre tres semanas después de que los síntomas encontraran opacidad de parches pulmonares con engrosamiento de las paredes bronquiales y engrosamiento del tabique intralobulillario e interlobulillario ${ }^{4}$. En la mayoría de los pacientes con opacidad en vidrio pulido se han encontrad posteriormente fibrosis ${ }^{5}$.

El diagnóstico de COVID-19 depende de estudios por imágenes, como tomografía torácica simple, que permite identificar daños típicos, y clasificar según la clasificación CO-RADS (COVID-19 Reporting and Data System) (Tabla 1),creada por la Asociación Holandesa de Radiólogos (NVVR).

Los hallazgos de tomografía según la clasificación CO-RADS proporcionan un nivel de sospecha de afectación pulmonar de COVID-19, aumentando el nivel de sospecha muy baja (CO-RADS 1) a sospechas muy altas (CO-RADS 5), además de identificar tomografía insuficiente (CO-RADS 0) o una tomografía positiva más prueba PCR positiva (CO-RADS 6). A CO-RADS 0 no se le puede asignar ninguna de las cinco categorías debido a exploraciones tomográficas incompletas o de bajo calidad. El CO-RADS 1 es como un nivel muy bajo de sospecha de COVID-19, CO-RADS 2 implica una baja afectación pulmonar sospechosa basada en hallazgos de TC. Se pueden encontrar características de patrón nodular centrilobular, consolidación lobular o segmental y cavitación pulmonar. CO-RADS 3 implica hallazgos de COVID19, características de neumonía viral o etiologías no infecciosas como vidrio pulido perihiliano, o vidrio pulido con espesamiento de tabique interlobulillario liso con o sin derrame pleural. CO-RADS 4 tiene características típicas de COVID-19, los hallazgos son como otra neumonía viral, que es que hay inflamación peribroncovascular. CO-RADS 5 tiene un nivel muy alto de COVID-19 sospechoso con hallazgos típicos de tomografía computarizada (TC), así como opacidad de vidrio pulido, con o sin consolidaciones, en superficies pleurales viscerales? 
Tabla 1. Principales hallazgos radiológicos en tomografía torácica según la clasificación CO-RADS (COVID-19 Reporting and Data System)

Descripción
Bajo nivel de sospecha
TC normal
TC sin infección
CO-RADS 1

Bajo nivel de sospecha TC sin datos de infección por SARS-CoV-2

Patrón en árbol de gemación Patrón nodular centrolobulillar Consolidación lobar y segmentada Cavitación pulmonar

\section{CO-RADS 3}

TC con hallazgos de neumonía viral

Vidrio despulido perihiliar, vidrio despulido con engrosamiento septal interlobulillar, edema pleural Radioopacidad en vidrio despulido Radioopacidad en vidrio despulido lejos de las pleuras

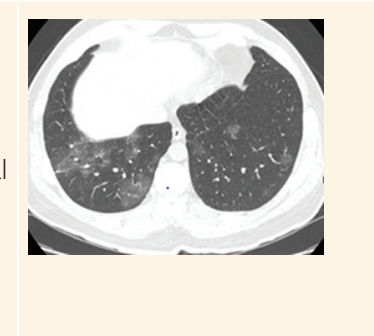

CO-RADS 4

Alto nivel de sospecha, puede sobreponerse a una neumonía viral

Algunas características del CO-RADS 5 sin contacto de pleura visceral, unilateral estrictamente

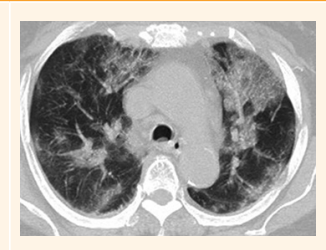

\section{CO-RADS 5}

Muy alto nivel de sospecha Patrón en vidrio despulido con o sin radioopacidades

Radioopacidad bilateral o multifocal, cercano a la pleura visceral, incluyendo fisuras

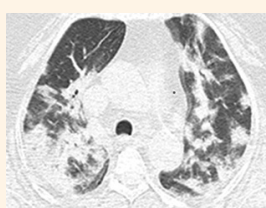

TC: tomografía computarizada; SARS-CoV-2: coronavirus 2 del síndrome respiratorio agudo grave.

Adaptada de Pan, et al., $2020^{5}$.

El uso de tomografía por emisión de positrones/ tomografía computarizada con fluorodesoxiglucosa (FDG-PET/CT) puede ser útil para confirmar la infección por SARS-CoV-2 ${ }^{8}$. El diagnóstico definitivo se realiza mediante reacción en cadena de la polimerasa con transcripción inversa en tiempo real (RT-PCR) en muestras obtenidas de hisopos orofaríngeos $y$ nasofaríngeos. Este estudio tiene una sensibilidad del 59 al $71 \%$ en comparación con las pruebas repetidas ${ }^{9}$.

Aunque el hallazgo típico de TC COVID-19 se observa en pacientes confirmados, estas características se pueden encontrar en pacientes con un resultado inicial falso negativo RT-PCR ${ }^{10}$.

Los pacientes con CO-RADS 5, linfopenia, ferritina sérica alta, proteína $C$ reactiva, dímero $D$ y lactato deshidrogenasa se encontraron en formas graves de COVID-19. Además de tener una proteína $C$ reactiva $>10 \mathrm{mg} / \mathrm{l}$ y linfopenia inferior a $0.8 \times 10^{9} / \mathrm{I}^{11}$.

En población china el rango de edad en pacientes diagnosticados con COVID-19 que desarrollaron síntomas graves era de 52-66 años ${ }^{12,13}$. En la población italiana, la tasa de mortalidad aumenta con la edad en aproximadamente un $12 \%$ en pacientes mayores de 70 años y un $20 \%$ en los mayores de 80 años $^{14}$. Algunos estudios señalan que el sexo masculino es el más afectado, y la fiebre, la tos y la disnea fueron los principales síntomas de la enfermedad ${ }^{15}$.

Algunos informes mencionan que la mayoría de los pacientes con COVID-19 tienen diabetes mellitus, hipertensión y enfermedades cardiacas de diagnóstico previo ${ }^{16}$.

La medida de la proteína $C$ reactiva refleja la magnitud del síndrome inflamatorio sistémico en formas graves, produciendo una «tormenta de citocinas» que conduce a daños agudos en los tejidos y posterior falla multisistema ${ }^{17}$. Las altas tasas de mortalidad de los pacientes de cuidados intensivos se han visto en algunos informes de China, redondeadas del 81 al $97 \%{ }^{18}$. Además, algunos informes mencionan una relación entre la COVID-19 y lesión renal aguda que aumenta la mortalidad intrahospitalaria ${ }^{19}$.

Algunos informes han mencionado que la TC tiene una especificidad inferior al 50\%, sin embargo, los estudios actuales sugieren que esta especificidad es aún mayor ${ }^{20,21}$.

Debido a que la sensibilidad de la RT-PCR disminuye después de siete días de síntomas, la TC sugiere diagnosticar COVID-19 en pacientes con síntomas más duraderos ${ }^{22}$. La clasificación de CO-RADS de uso puede detectar a más del $95 \%$ de los pacientes con síntomas respiratorios, e incluso una puntuación de CO-RADS $\geq 4$ se asoció con un diagnóstico preciso de COVID-1923.

La clasificación de la Sociedad Radiológica de América del Norte (RSNA) es muy similar a la clasificación CO-RADS, lo que permite el uso de ambos sistemas con casi los mismos resultados ${ }^{24}$. 


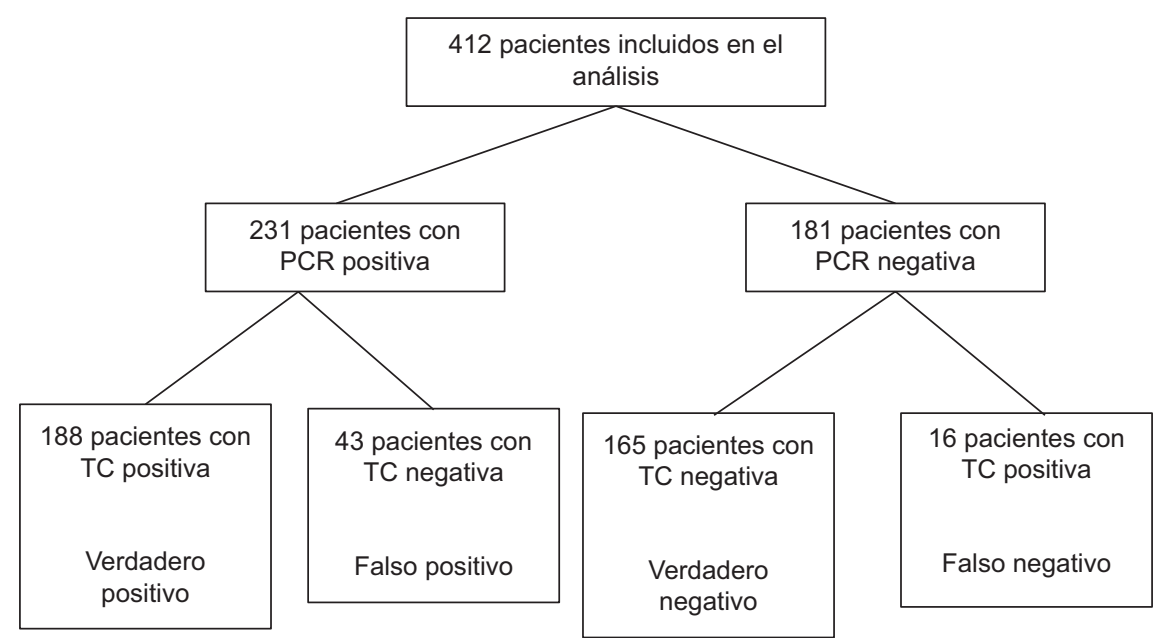

Figura 1. Diagrama de flujo de pacientes de estudio, con pacientes con reacción en cadena de la polimerasa con transcripción inversa en tiempo real (RT-PCR) positivos y negativos, y pacientes con tomografía computarizada (TC) positivos y negativos.

La presencia de obesidad y presión arterial alta tiene una asociación significativa con enfermedades graves, mostrando un quirófano de 6.49 , lo que sugiere una interacción sinérgica entre ellos. Además, hay un mayor riesgo de progresión a COVID-19 grave y un tiempo de propagación viral más largo en pacientes con enfermedad del hígado graso no alcohólico ${ }^{25}$. Por otra parte, se han descrito otros estudios de imagen con gran utilidad, siendo el ultrasonido uno de ellos, mostrando ventajas como ausencia de exposición a la radiación ionizante para los trabajadores de la salud, siendo una técnica diagnóstica segura, rápida y no invasiva que se utiliza junto a la cama del paciente. Por lo tanto, la ecografía pulmonar es una alternativa eficaz para diagnosticar y monitorear a los pacientes con neumonía por COVID-1926.

\section{Material y métodos}

Buscamos todos los resultados de RT-PCR de pacientes de nuestra unidad, con diagnóstico sospechoso o confirmado, de marzo de 2020 a diciembre de 2020, luego lo buscamos TC torácicas de pacientes con prueba previa de PCR, incluyendo pacientes mayores de 18 años de edad, pacientes con neumonía atípica sospechosa o confirmada por SARS-CoV-2; excluyendo a los pacientes con neumonía viral causada por otros que el SARS-CoV-2, y a los pacientes que no tienen un diagnóstico definitivo de neumonía por el SARS-CoV-2. A continuación, todas las TC se clasificaron según criterios CO-RADS para determinar el diagnóstico probable o confirmado de COVID-19. EI análisis estadístico se realizó utilizando SPSS versión 26 y Minitab versión 18. Las variables nominales se probaron mediante las pruebas chi cuadrada de Pearson. El rendimiento diagnóstico de la TC torácica se evaluó como precisión diagnóstica, sensibilidad, especificidad, valor predictivo positivo y valor predictivo negativo, tomando como referencia el resultado RT-PCR más cercano a la TC (Fig. 1). Los días de hospitalización fueron analizados en un gráfico de supervivencia utilizando un modelo Kaplan-Meier.

\section{Resultados}

Obtuvimos 412 pacientes con RT-PCR y TC torácica, incluidos 213 pacientes con RT-PCR positiva, y 181 pacientes con RT-PCR; de 213 pacientes con RT-PCR positivo, 188 pacientes tuvieron TC torácica positiva para neumonía por SARS-CoV-2 y 43 pacientes con TC torácica negativa para neumonía por SARS-CoV-2; de 181 pacientes con RT-PCR negativa, 165 pacientes tuvieron TC torácica negativa para neumonía por SARS-CoV-2, y 16 pacientes con TC torácica positiva para neumonía por SARS-CoV-2. Las principales RT-PCR positivas pertenecen a personas de 44 a 69 años, y las RT-PCR negativas pertenecen a personas de 49 a 73 años, aumentando la mortalidad en el sexo masculino durante seis días de estancia hospitalaria (Fig. 2). Obtuvimos una sensibilidad del $92.15 \%$ y una especificidad del $79.32 \%$, estos asociados principalmente a pacientes con mal pronóstico que se hicieron con TC torácica. Además, la mortalidad aumenta en el grupo CO-RADS 4,5 y 6 , 


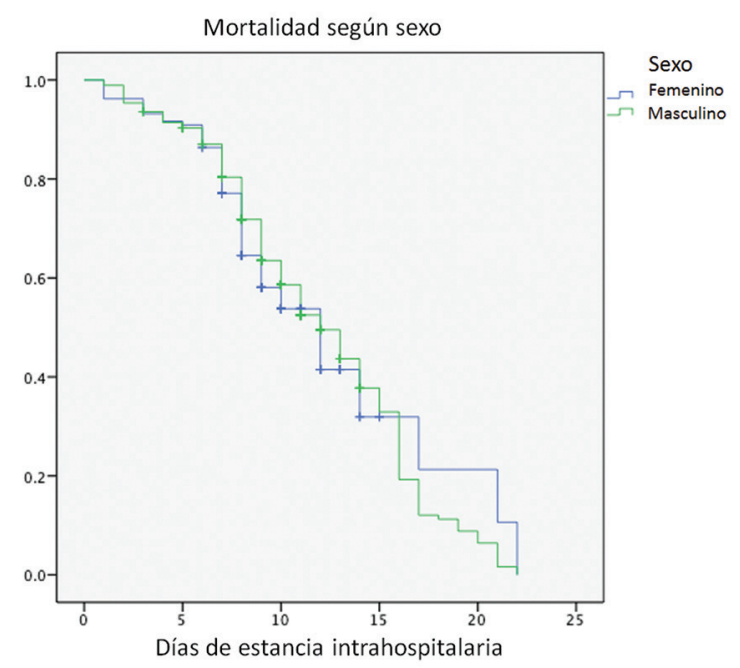

Figura 2. Gráfico de Kaplan-Meier que muestra la mortalidad a lo largo de días de hospitalización según el sexo.

Mortalidad según el estadio CO-RADS

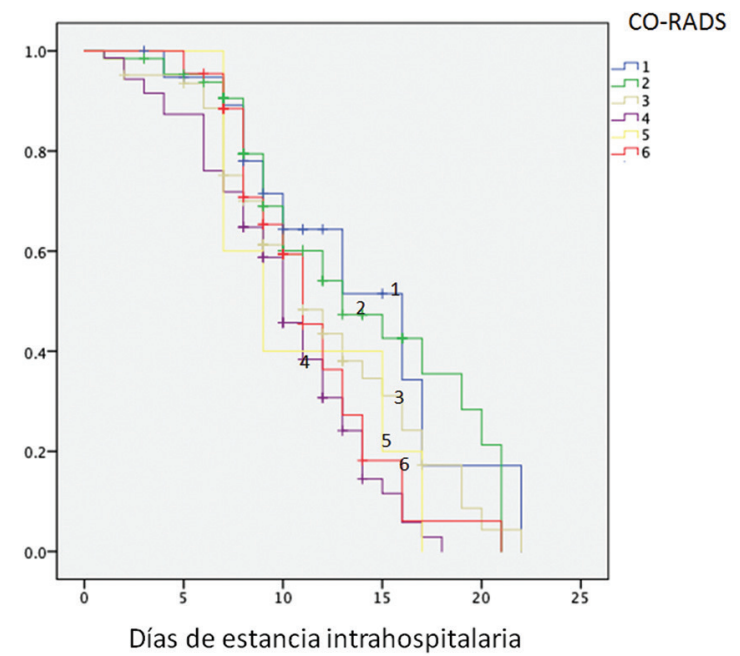

Figura 3. Gráfico de Kaplan-Meier que muestra la mortalidad a lo largo de días de hospitalización según la clasificación de CO-RADS (COVID-19 Reporting and Data System).

en comparación con CO-RADS 1, 2 y 3, sin embargo, se encontró una estancia de hospitalización más larga en el grupo CO-RADS 1, 2 y 3 (Fig. 3).

\section{Discusión y conclusión}

La COVID-19 tiene alta transmisibilidad, aumentando el riesgo de transmisión en comunidad, lo que explica la alta propagación del virus en nuestro entorno ${ }^{27}$. El diagnóstico precoz desempeña un papel decisivo para controlar la propagación de la COVID19; la RT-PCR es el estándar de oro actual; sin embargo, el método principal utilizado en nuestras instituciones, los falsos negativos pueden ser comunes cuando se utilizan hisopos orales; razón por la cual se deben adoptar múltiples métodos de detección para confirmar el diagnóstico de COVID-19, ganando importancia el uso de la TC torácica, con una alta sensibilidad y especificidad para las manifestaciones pulmonares, incluso en las primeras eta$\operatorname{pas}^{27,28}$. Esta herramienta es rápida y accesible para la mayoría de las personas en un centro de segundo o tercer nivel en nuestro país, y útil para detectar COVID-19, especialmente en RT-PCR negativa falsa. La TC torácica puede ayudar a confirmar o descartar casos sospechosos de COVID-19, clasificarlos en el sistema CO-RADS y como una herramienta de pronóstico. Otros estudios en nuestro país muestran valores de sensibilidad y especificidad más cercanos a nuestros hallazgos, como Velasco, et al., que obtuvo una sensibilidad de la TC del $89 \%$ y especificidad del $66 \%$, sin embargo, evaluaron menos pacientes que nuestro estudio, lo que podría explicar la diferencia entre sensibilidad y especificidad de nuestros estudios.

\section{Financiamiento}

No hubo financiamiento para este trabajo.

\section{Conflicto de intereses}

Los autores declaran no tener ningún conflicto de intereses.

\section{Responsabilidades éticas}

Protección de personas y animales. Los autores declaran que para esta investigación no se han realizado experimentos en seres humanos ni en animales.

Confidencialidad de los datos. Los autores declaran que han seguido los protocolos de su centro de trabajo sobre la publicación de datos de pacientes.

Derecho a la privacidad y consentimiento informado. Los autores han obtenido el consentimiento informado de los pacientes y/o sujetos referidos en el artículo. Este documento obra en poder del autor de correspondencia.

\section{Bibliografía}

1. Lu R, Zhao X, Li J, Niu P, Yang B, Wu H, et al. Genomic characterization and epidemiology of 2019 novel coronavirus: implications for virus origins and receptor binding. Lancet. 2020;395(10224):565-74. 
2. The novel coronavirus pneumonia emergency response epidemiology team. Vital surveillances: the epidemiological characteristics of an outbreak of 2019 novel coronavirus diseases (COVID-19). China, 2020. China CDC Weekly. 2020;2(8):113-22.

3. Song F, Shi N, Shan F, Zhang Z, Shen J, Lu H. Emerging coronavirus (2019-nCoV) pneumonia. Radiology. 2020;295(1):210-7.

4. Jin YH, Cai L, Cheng ZS, Cheng H, Deng T, Fan YP. A rapid advice guideline for the diagnosis and treatment of 2019 novel coronavirus (2019-nCoV) infected pneumonia (standard version). Mil Med Res. 2020;7(1):4.

5. Pan Y, Guan H, Zhou S, Wang Y, Li Q, Zhu T. Initial CT findings and temporal changes in patients with the novel coronavirus pneumonia (2019-nCoV): A study of 63 patients in Wuhan, China. Eur Radiol. 2020;30(6):3306-9.

6. Prokop M, van Everdingen W, van Rees Vellinga T, Quarles van Ufford J, Stoger L, Beenen L, et al. CO-RADS - A categorical CT assessment scheme for patients with suspected COVID-19: definition and evaluation. Radiology. 2020;296(2):E97-E104.

7. Simpson S, Kay FU, Abbara S, Bhalla S, Chung JH, Chung M, et al. Radiological Society of North America Expert Consensus Statement on Reporting Chest CT Findings Related to COVID-19. Endorsed by the Society of Thoracic Radiology, the American College of Radiology, and RSNA. Radiol Cardiothorac Imaging. 2020;2(2):e200152.

8. Maurea S, Mainolfi CG, Bombace C, Annunziata A, Attanasio L, Petretta M, et al. FDG-PET/CT imaging during the Covid-19 emergency: a southern Italian perspective. Eur J Nucl Med Mol Imaging. 2020;47(11):2691-7.

9. Pang J, Wang MX, Ang IYH, Tan SHX, Lewis RF, Chen Jl, et al. Potential rapid diagnostics, vaccine and therapeutics for 2019 novel coronavirus (2019-nCoV): a systematic review. J Clin Med. 2020;9(3):623.

10. Xie X, Zhong Z, Zhao W, Zheng C, Wang F, Liu J. Chest CT for typical 2019-nCoV pneumonia: relationship to negative RT- PCR testing. Radiology. 2020;296(2):E41-E45.

11. El Aidaoui K, Haoudar A, Khalis M, Kantri A, Ziati J, El Ghanmi A, et al Predictors of severity in Covid-19 patients in Casablanca, Morocco. Cureus. 2020;12(9):e10716.

12. Wang D, Hu B, Hu C, Zhu F, Liu X, Zhang J, et al. Clinical characteristics of 138 hospitalized patients with 2019 novel coronavirus-infected pneumonia in Wuhan, China. JAMA. 2020;323(11):1061-9.

13. Yang $X, Y u Y, X u J$, Shu $H$, Xia J, Liu H, et al. Clinical course and outcomes of critically ill patients with SARS-CoV-2 pneumonia in Wuhan China: a single-centered, retrospective, observational study. Lancet Respir Med. 2020;8:475-81.

14. Livingston E, Bucher K. Coronavirus disease 2019 (COVID-19) in Italy. JAMA. 2020;323:1335.
15. Zheng Z, Peng F, Xu B, Zhao J, Liu H, Peng J, et al. Risk factors of critical \& mortal COVID-19 cases: a systematic literature review and meta-analysis. J Infect. 2020;81:e16-e25.

16. Huang $\mathrm{C}$, Wang $\mathrm{Y}$, Li X, Ren L, Zhao J, Hu Y, et al. Clinical features of patients infected with 2019 novel coronavirus in Wuhan, China. Lancet. 2020;395:497-506

17. Henry BM, de Oliveira MHS, Benoit S, Plebani M, Lippi G. Hematologic, biochemical and immune biomarker abnormalities associated with severe illness and mortality in coronavirus disease 2019 (COVID-19): a meta- analysis. Clin Chem Lab Med. 2020;58:1021-8.

18. Liu W, Tao Z-W, Wang L, Yuan ML, Liu K, Zhou L, et al. Analysis of factors associated with disease outcomes in hospitalized patients with 2019 novel coronavirus disease. Chin Med J (Engl). 2020;133:1032-8.

19. Cheng Y, Luo R, Wang K, Zhang M, Wang Z, Dong L, et al. Kidney disease is associated with in-hospital death of patients with COVID-19. Kidney Int. 2020;97:829-38.

20. Kim H, Hong H, Yoon SH. Diagnostic performance of CT and reverse transcriptase-polymerase chain reaction for coronavirus disease 2019: A meta-analysis. Radiology. 2020;296(3):E145-E155.

21. Himoto $Y$, Sakata A, Kirita M, Hiroi T, Kobayashi KI, Kubo K, et al. Diagnostic performance of chest CT to differentiate COVID-19 pneumonia in non-high-epidemic area in Japan. Jpn J Radiol. 2020;38(5):400-6.

22. Tahamtan A, Ardebili A. Real-time RT-PCR in COVID-19 detection: issues affecting the results. Expert Rev Mol Diagn. 2020;20(5):453-4.

23. Schalekamp S, Bleeker-Rovers CP, Beenen LFM, Quarles van Ufford HME, Gietema HA, Stöger JL, et al. Chest CT in the Emergency Department for diagnosis of COVID-19 pneumonia: Dutch experience. Radiology. 2021;298(2):E98-E106.

24. Palomar-Lever A, Barraza G, Galicia-Alba J, Echeverri-Bolaños M, Escarria-Panesso R, Padua-Barrios J, et al. Hepatic steatosis as an independent risk factor for severe disease in patients with COVID-19: A computed tomography study. JGH Open. 2020;4(6):1102-7.

25. Zhang W, Du RH, Li B, Zheng XS, Yang XL, Hu B, et al. Molecular and serological investigation of 2019-nCoV infected patients: implication of multiple shedding routes. Emerg Microbes Infect. 2020;9:386-9.

26. Karacaer C, Karabay O, Gunduz Y, Yaylacı S, Guclu E. Correlation of lung ultrasound and computed tomography findings in COVID-19 pneumonia. J Coll Physicians Surg Pak. 2020;30(10):147-52.

27. Li T. Diagnosis and clinical management of severe acute respiratory syndrome coronavirus 2 (SARS-CoV-2) infection: an operational recommen- dation of Peking Union Medical College Hospital (V2.0). Emerg Microbes Infect. 2020;9:582-5.

28. Xie X, Zhong Z, Zhao W, Zheng C, Wang F, Liu J. Chest CT for typical 2019-nCoV pneumonia: relationship to negative RT-PCR testing. Radiology. 2020;296:E41-E45. 